\title{
Lessons learned from the delivery of virtual integrative oncology interventions in clinical practice and research during the COVID-19 pandemic
}

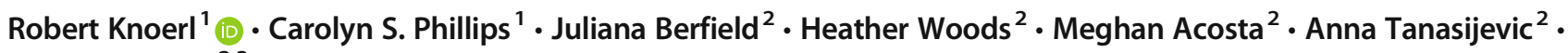 \\ Jennifer Ligibel ${ }^{2,3}$
}

Received: 30 January 2021 / Accepted: 21 March 2021 / Published online: 26 March 2021

(C) The Author(s), under exclusive licence to Springer-Verlag GmbH Germany, part of Springer Nature 2021

\begin{abstract}
s
The outbreak of the coronavirus disease 2019 (COVID-19) and subsequent need for disease transmission mitigation efforts have significantly altered the delivery of cancer care (e.g., rise of telemedicine), including within the field of integrative oncology. However, little has been described about how National Cancer Institute-Designated Cancer Centers have transformed integrative oncology care delivery in response to the COVID-19 pandemic. The purpose of this commentary is to describe the delivery of integrative oncology clinical services and conduct of research at The Leonard P. Zakim Center for Integrative Therapies and Healthy Living at Dana-Farber Cancer Institute during the COVID-19 pandemic. Clinical services transitioned from an array of in-person appointment-based services, such as acupuncture and massage, and group programs, such as yoga and nutrition seminars to a combination of live-streamed and on-demand virtual group programs and one-on-one virtual appointments for services such as acupressure and self-care massage. Group program volume grew from 2189 in-person program patient visits in the 6 months prior to onset of the COVID pandemic to 16,366 virtual (e.g., live-streamed or on-demand) patient visits in the first 6 months of the pandemic. From a research perspective, two integrative oncology studies, focused on yoga and music therapy, respectively, were transitioned from in-person delivery to a virtual format. Participant accrual to these studies increased after the transition to virtual consent and intervention delivery. Overall, our clinical and research observations at Dana-Farber Cancer Institute suggest that the delivery of virtual integrative oncology treatments is feasible and appealing to patients. Trial Registration: NCT03824860 (Yoga); NCT03709225 (Music Therapy)
\end{abstract}

Keywords Integrative oncology $\cdot$ Integrative medicine $\cdot$ Telemedicine $\cdot$ Symptom management

\section{Introduction}

The emergence of the coronavirus disease 2019 (COVID-19) in the USA has significantly altered healthcare practice and research implementation. The number of telehealth visits increased by approximately $154 \%$ when comparing telehealth

Robert Knoerl

Robert_knoerl@dfci.harvard.edu

1 Phyllis F. Cantor Center for Research in Nursing and Patient Care Services, Dana-Farber Cancer Institute, 450 Brookline Ave, LW518, Boston, MA 02215, USA

2 The Leonard P. Zakim Center for Integrative Therapies and Healthy Living, Dana-Farber Cancer Institute, Boston, MA, USA

3 Medical Oncology, Dana-Farber Cancer Institute, Boston, MA, USA visit frequency during the last week of March in 2019 and 2020, respectively [1]. Due to the need for social distancing to decrease the transmission of COVID-19, a portion of inperson oncology clinic visits have also been switched to telemedicine visits [2-4]. In addition, while oncology clinical trials have continued during the COVID-19 pandemic, patient enrollment to oncology clinical trials has slowed and investigators have had to identify alternative methods to continue research-related patient interactions $[4,5]$. For example, evidence suggests that up to $20 \%$ of cancer survivors $(N=933)$ are less likely to participate in oncology clinical trials due to fears associated with the COVID-19 pandemic [6].

Integrative oncology care delivery has also been significantly impacted by the COVID-19 pandemic. Approximately $70 \%$ of National Cancer Institute-Designated Cancer Centers offer integrative oncology treatments such as acupuncture, massage, and/or meditation [7]. Integrative 
medicine typically includes personalized "high-touch" services, such as acupuncture and massage, as well as group programs in yoga, music, and other modalities, making a transition to remotely delivered care challenging. Even before the onset of the COVID-19 pandemic, the use of technology (e.g., mobile health [8] or telephone [9]) to support cancer treatment-related symptom management (e.g., depression, anxiety, fatigue) [9] has been on the rise. However, with the exception of exercise-related studies [8], most previously tested mobile health or telephone-based oncology symptom management approaches have not focused on integrative oncology interventions [9]. To this point, little has been described about how integrative oncology services offered by National Cancer Institute-Designated Cancer Centers have been transformed in response to the COVID-19 pandemic. Within this commentary, we describe how integrative oncology care delivery in clinical practice and research at The Leonard P. Zakim Center for Integrative Therapies and Healthy Living was altered in response to the pandemic.

\section{Clinical practice}

The Leonard P. Zakim Center for Integrative Therapies and Healthy Living is an integrative oncology center at DanaFarber Cancer Institute with a joint mission of providing evidence-based integrative oncology services and healthy living interventions to patients during and after cancer treatment and of advancing the science of integrative oncology through research. The Zakim Center is staffed by a team of physicians, therapists (e.g., yoga, exercise, music), nurses, and other healthcare professionals and offers individual appointmentbased services such as acupuncture and massage and group programs in areas such as yoga, exercise, nutrition, meditation, and expressive arts. Prior to the COVID-19 pandemic, most appointments and group programs were offered in person, through The Zakim Center Clinic, on Dana-Farber's main campus.

The Zakim Center Clinic closed to all appointments and programs in mid-March 2020 to limit patient and staff exposure and to reduce total patient and staff volume on campus. Although the goal was to restore in-person acupuncture and massage appointments as quickly as deemed safe, and these services were resumed in late summer, it was clear that inperson group programming would not be feasible for an indefinite period of time. The Zakim Center thus launched livestreamed virtual classes in yoga, meditation, strength training, and nutrition in late March. Additional class offerings and a library of previously recorded programs available for viewing on-demand were added over the summer. Patient uptake of virtual programs was significant; The Zakim Center provided 16,366 patient visits, including 6383 visits to live-streamed programs and 9983 visits to on-demand programming in the first 6 months of the COVID-19 pandemic, as compared to 2189 patient visits to in-person group programming in the 6 months prior to the COVID-19 pandemic. The virtual offerings most attended by patients included exercise (yoga, strength training, Tai Chi, Qigong), followed by meditation and mindfulness, nutrition, and expressive arts therapy. The frequency of participant visits by program type for the inperson, virtual, and on-demand class offerings before and during the COVID-19 pandemic is described in Fig. 1.

\section{Research}

Two integrative oncology research studies were ongoing at The Zakim Center prior to the onset of the COVID-19 pandemic. The first trial was designed to evaluate the feasibility and benefits of a mindfulness-based music therapy intervention in adolescents and young adults receiving cancer treatment. The original program included four 45-min in-person mindfulness-based music therapy sessions with a boardcertified music therapist during chemotherapy infusions. The second trial was designed to evaluate the feasibility and benefits of an 8-week yoga intervention for cancer survivors with chronic painful chemotherapy-induced peripheral neuropathy and originally included in-person (i.e., attend one 45-min group yoga class per week at The Zakim Center) and homebased yoga classes. In June 2020, both studies were amended to allow telephone-based recruitment and informed consent, data collection via email, and remote delivery of the mindfulness-based music therapy and yoga interventions. After these changes were activated, the recruitment rate for both studies increased from $\sim 2.6$ participants/month to $\sim 2.83$ (music therapy study) and $\sim 4.83$ (yoga study) participants/ month.

\section{Conclusion}

Our preliminary clinical and research observations suggest that patients are interested in the virtual delivery of integrative oncology interventions, and such interventions are feasible to implement. Uptake of group programs and study enrollment for integrative oncology interventions increased when interventions were switched from in-person to virtual. The virtual interventions may have been more appealing to participants because the virtual format addressed potential barriers to participants' uptake of integrative oncology interventions, such as travel, lack of knowledge about the availability of the services, or conflicting cancer therapy appointments [10]. Further research will be needed to determine equivalence in efficacy between virtual and in-person integrative oncology intervention formats for varying symptoms. The implementation of feasible and cost-effective methods to deliver integrative 


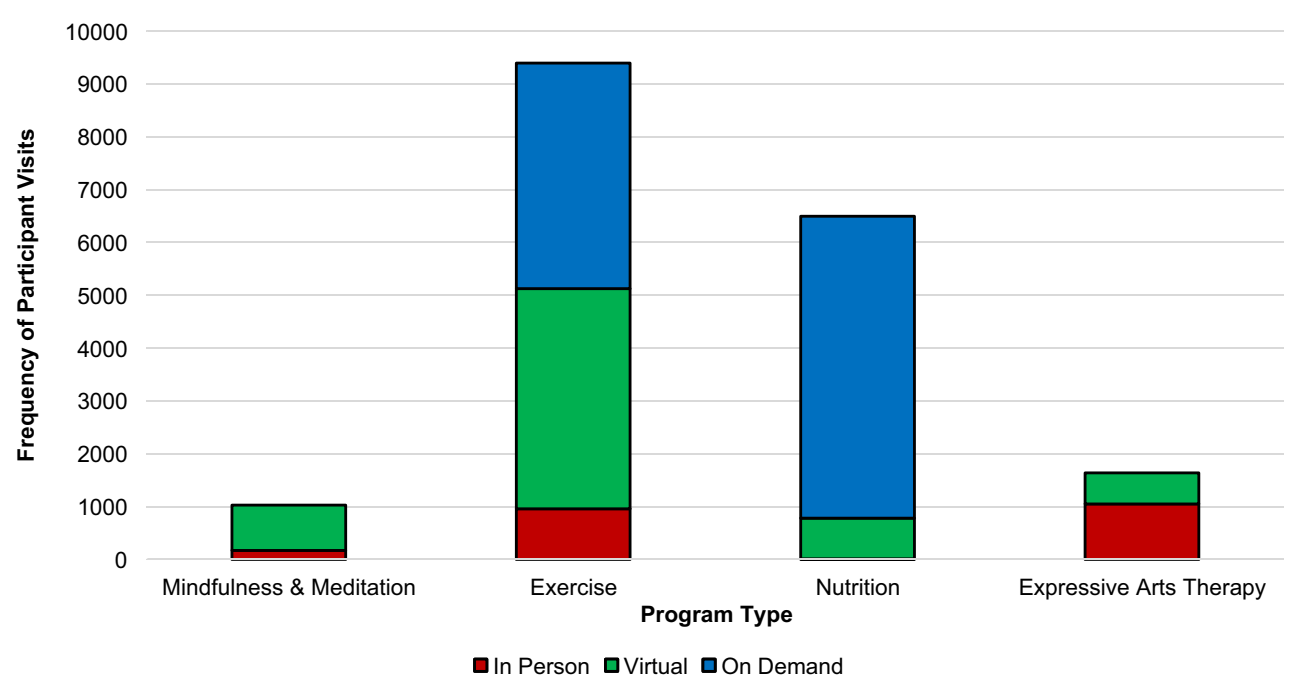

Fig. 1 Frequency of participant visits by program type at The Leonard P. Zakim Center for Integrative Therapies and Healthy Living at DanaFarber Cancer Institute before and during the COVID-19 pandemic. Figure 1 describes the frequency of participant visits by program type at The Zakim Center from October 2019 to March 22, 2020 (in-person class offerings only), and March 23, 2020, to September 30, 2020 (virtual and

oncology symptom management interventions for oncology patients will be critical not only during the COVID-19 pandemic, but to increase the availability of these interventions to oncology patients in the future.

Acknowledgements We would like to acknowledge Erica Fox, Hang Phan, Raymond Lamothe, Kelsey Maymon, and Barbara Halpenny for their assistance in participant recruitment, participant data collection, and/ or study management. We would also like to acknowledge Lauren Orsillo, Tom Leu, Sara Menendez, and Kelly Chisholm for their assistance in scheduling the virtual appointments for both studies.

Availability of data and material The datasets used and/or analyzed during the current study are available from the corresponding author on reasonable request.

Author contribution RK, HW, MA, JB, CP, AT, and JL were all involved in the design of the commentary, interpretation of the data, and writing of the manuscript. All authors have read and approved the final manuscript.

Funding Part of the work described in this manuscript (NCT03824860) was supported by the Oncology Nursing Foundation.

\section{Declarations}

Ethics approval This study was performed in line with the principles of the Declaration of Helsinki. Approval was granted by the Dana-Farber/ Harvard Cancer Center Office for Human Research Studies (Protocols: $18-341 \& 18-578)$.

Consent to participate Informed consent was obtained from all individual participants included in either study.

Consent for publication Not applicable on-demand class offerings only). Note: Exercise program offerings included classes such as yoga, strength training, Tai Chi, and Qigong. Expressive arts therapy program offerings included classes such as creative arts workshops and music therapy. Meditation and mindfulness program offerings included classes such as mind body resilience and mindfulness meditation

Conflict of interest Dr. Knoerl reports consulting honorariums from the Strategy Inc., Spark Healthcare, and System Analytic, outside the submitted work. The other authors have no conflicts of interest to declare that are relevant to the content of this article.

\section{References}

1. Koonin LM, Hoots B, Tsang CA et al (2020) Trends in the use of telehealth during the emergence of the COVID-19 pandemic United States, January-March 2020. MMWR Morb Mortal Wkly Rep 69:1595-1599. https://doi.org/10.15585/mmwr.mm6943a3

2. Meti N, Rossos PG, Cheung MC, Singh S (2020) Virtual cancer care during and beyond the COVID-19 pandemic: We Need to Get It Right. JCO Oncol Pract 16:527-529. https://doi.org/10.1200/op. 20.00281

3. Mulvey TM, Jacobson JO (2020) Covid-19 and cancer care: Ensuring safety while transforming care delivery. J Clin Oncol 38:3248-3251

4. Mullangi S, Schleicher SM, Aviki EM (2020) Innovation in cancer care delivery in the era of COVID-19. JCO Oncol Pract OP 20:336. https://doi.org/10.1200/op.20.00336

5. Waterhouse DM, Harvey RD, Hurley P, Levit LA, Kim ES, Klepin HD, Mileham KF, Nowakowski G, Schenkel C, Davis C, Bruinooge SS, Schilsky RL (2020) Early impact of COVID-19 on the conduct of oncology clinical trials and long-term opportunities for transformation: findings from an American Society of Clinical Oncology Survey. JCO Oncol Pract 16:417-421. https://doi.org/10. 1200/op.20.00275

6. Fleury ME, Farner AM, Unger JM (2020) Association of the COVID-19 outbreak with patient willingness to enroll in cancer clinical trials. JAMA Oncol 7:131-132

7. Yun H, Sun L, Mao JJ (2017) Growth of integrative medicine at leading cancer centers between 2009 and 2016: a systematic analysis of NCI-designated comprehensive cancer center websites. J Natl Cancer Inst - Monogr 2017:29-32. https://doi.org/10.1093/ jncimonographs/lgx004 
8. Chan RJ, Howell D, Lustberg MB, Mustian K, Koczwara B, Ng CC, Kim Y, Nápoles AM, Dixit N, Klemanski D, Ke Y, Toh YL, Fitch MI, Crichton M, Agarawal S, Chan A (2020) Advances and future directions in the use of mobile health in supportive cancer care: proceedings of the 2019 MASCC Annual Meeting symposium. Support. Care Cancer 28:4059-4067

9. Ream E, Hughes AE, Cox A et al (2020) Telephone interventions for symptom management in adults with cancer. Cochrane Database Syst Rev 2020
10. Latte-Naor S, Mao JJ (2019) Putting integrative oncology into practice: concepts and approaches. J Oncol Pract 15:7-14

Publisher's note Springer Nature remains neutral with regard to jurisdictional claims in published maps and institutional affiliations. 